Journal of

Molecular Microbiology

and Biotechnology

\title{
Chromosome Architecture and Segregation in Prokaryotic Cells
}

\author{
Peter L. Graumann \\ SYNMIKRO, LOEWE Center for Synthetic Microbiology, and Department of Chemistry, Philipps University Marburg, \\ Marburg, Germany
}

The 3-D structure of prokaryotic chromosomes, their copy number, replication and segregation have gained an enormous interest in the past 15 years, for the most part because the spatial dynamics of chromosomes have become amenable for analysis through fluorescence microscopy techniques and other single-cell-based assays. And, of course, because these issues are of fundamental biological importance and impact on many cellular processes such as global gene regulation and the cell cycle.

This special issue of Journal of Molecular Microbiology and Biotechnology deals with various aspects of bacterial and archaeal chromosomes; their arrangement, their dynamics and segregation mechanisms. Several reviews in this issue deal with different aspects of chromosome organization and partitioning in selected model bacteria, and processes such as dimer resolution and important factors such as nucleoid-associated proteins (NAPs), SMC proteins and topoisomerases are discussed in greater detail in the following chapters. Bacteria can have vastly different numbers of their chromosome, which is discussed in one chapter [Soppa (pp. 409-419)], the domain structure of the Escherichia coli chromosome [Messerschmidt and Waldminghaus (pp. 301-315)], and the dynamics of the two chromosomes in Vibrio cholerae [Ramachandran et al. (pp. 360-370)] have received their own chapters, because considerable knowledge has accumu-

\section{KARGER}

E-Mail karger@karger.com

www.karger.com $/ \mathrm{mmb}$ lated about these model organisms. This review is intended to provide an overview of the mode of chromosome segregation in bacteria in general and on what is known about chromosome dynamics in archaeal cells [see review by Samson and Bell (pp. 420-427)] and to give information on some groups of bacteria that have been studied in greater detail.

In bacteria such as E. coli and Bacillus subtilis, chromosomal DNA does not extend throughout the entire cytoplasm, as is the case for Caulobacter crescentus, but is condensed into a structure called the nucleoid (fig. 1). Compaction is mediated through nonspecific DNA-binding NAPs (which however do have a preference for certain DNA structures) [reviews by Dorman and van der Valk et al., pp. 316-331 and 344-359, respectively] and SMC proteins [Graumann and Knust, 2009]. The separation of duplicated chromosomes is extremely robust: only 1 in 10,000 cell cycle events shows a failure in DNA partitioning in B. subtilis, even when cells contain multiple replication forks and contain multiple chromosome copies [Ireton et al., 1994]. It is also possible to integrate an entire cyanobacterial chromosome (3.5 Mbp) into the B. subtilis chromosome $(4.2 \mathrm{Mbp})$, and cells still grow fairly well [Itaya et al., 2005], revealing great plasticity of a bacterial genome. A dedicated segregation motor has remained elusive; ParA proteins in C. crescentus [Ptacin et al., 2010]
(C) 2015 S. Karger AG, Basel

1464-1801/15/0246-0291\$39.50/0
Peter L. Graumann

SYNMIKRO, LOEWE Center for Synthetic Microbiology

Hans-Meerwein-Strasse

DE-35043 Marburg (Germany)

E-Mail peter.graumann@synmikro.uni-marburg.de 
and in V.cholerae [Fogel and Waldor, 2006] provide directionality of the segregation process [Ramachandran et al., pp. 360-370]. Inversions of smaller regions on the chromosome are not problematic, but the inversion of an entire arm of the E. coli chromosome results in slow growth, generation of anucleate cells, and mispositioning of chromosome regions (see below) [Niki et al., 2000]. This is in part due to the fact that normally, DNA and RNA polymerase frequently collide, because most genes are arranged such that transcription runs away from origin regions, as does replication. Upon inversion of e.g. a quarter of the $B$. subtilis chromosome, DNA polymerase movement is greatly slowed down [Merrikh et al., 2011]. Moreover, bacterial chromosomes have several features, including distinct domains and asymmetrically distributed DNA motifs, which aid in the organization and segregation of the genome, as detailed in this issue.

Most amazing at the time of discovery, bacterial chromosomes are not random coils of DNA, but are arranged in a spatially well-defined manner. The common scheme in several bacterial species is the observation that all loci on the chromosome have a rather defined position within the cell, and that chromosomes are arranged according to their physical structure, so that genes adjacent to each other on the chromosome will also be in close spatial proximity [Niki et al., 2000; Teleman et al., 1998; Viollier et al., 2004] (fig. 1). Factors such as SMC proteins are important for the maintenance of global chromosome arrangement, and are dealt with in two reviews within this compendium [Kleine Borgmann and Graumann, pp. 384-395, and Rybenkov et al., pp. 371-383]. Within the chromosome, relatively independent domains exist that are between 24 and $400 \mathrm{kbp}$ (dependent on the method used), and isolated against neighboring domains. Inside such topological domains, genes show close connections in terms of superhelical density and recombination events [Le et al., 2013; Postow et al., 2004; Staczek and Higgins, 1998]. These domains are thought to be composed of plectonemes, i.e. loops of superhelically twisted DNA, whose formation depends on several factors: (a) active transcription: the borders of chromosome interaction domains (CIDs) in C. crescentus are often flanked by highly expressed genes, whose translocation changes CID borders, and whose transcriptional inhibition leads to a major loss of CID formation; (b) condensation activity of SMC and NAPs, and (c) supercoiling, as CID borders become fuzzy during inhibition of topoisomerases [Le et al., 2013]. The existence of topologically isolated chromosome domains is quite handy because a DNA doublestrand break would not lead to a genome-wide relaxation

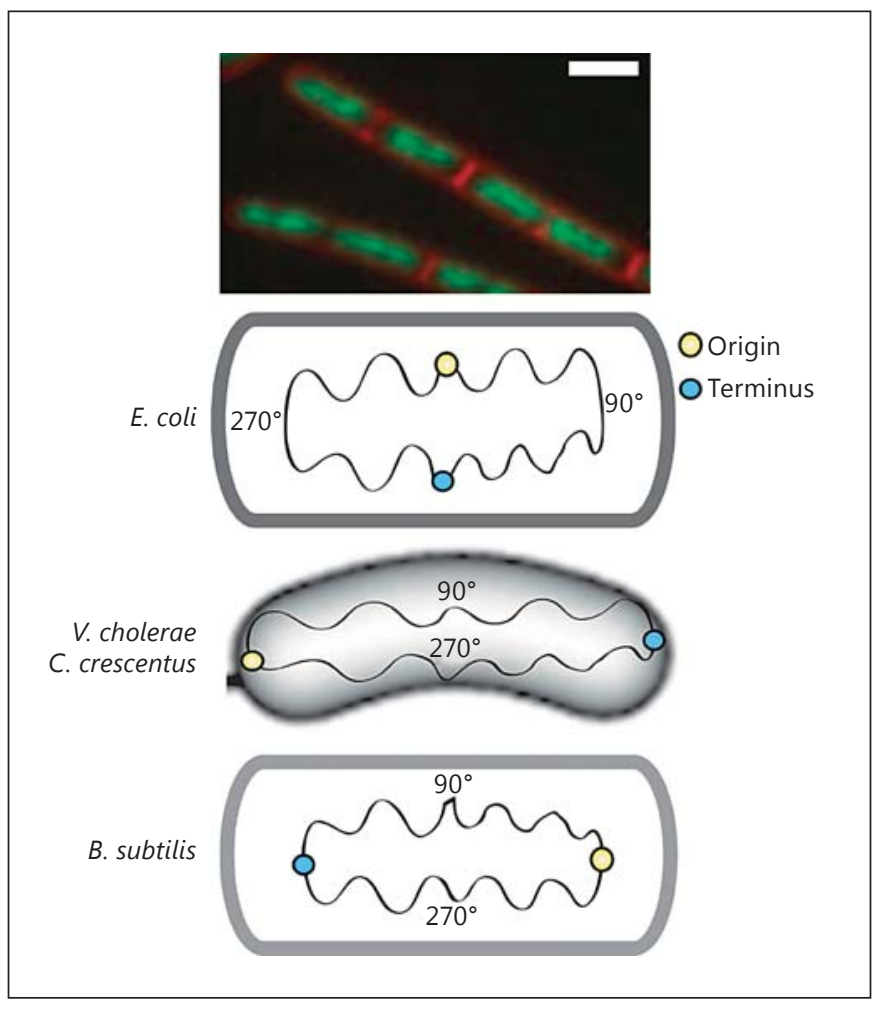

Fig. 1. Arrangement of bacterial chromosomes. Upper panel: fluorescence microscopy of chromosomes (stained in green) and cell membranes (stained in red). White bar $=2 \mu \mathrm{m}$. Drawings illustrate the arrangement of chromosomes in E. coli, V. cholerae (note that this organism has a second chromosome, which appears to be similar to a 'bacillus-like' chromosome), C. crescentus and B. subtilis. Origins $\left(0^{\circ}\right)$ and termini $\left(180^{\circ}\right)$ are illustrated as circles. Numbers indicate other positions on the circular chromosome.

of supercoils, but only affects a limited region of the chromosome. The domain structure of bacterial chromosomes may also facilitate segregation. In addition to CIDs, so-called 'macrodomains' exist, which can have a size of almost a quarter of the chromosome. Four such domains have been have been identified in E. coli, and they are again somewhat isolated from other macrodomains in terms of recombination events, subcellular positioning and segregation dynamics; these chromosomal superstructures are discussed in the reviews by Messerschmidt and Waldminghaus [pp. 301-315] and Muskhelishvili and Travers [pp. 332-343].

In general, chromosomes of mesophilic bacteria have overall negative supercoiling, with a superhelical density (sigma) of -0.06 (meaning $6 \%$ underwinding), facilitating the melting of the DNA duplex for transcription and replication initiation. Negative supercoiling is introduced by 


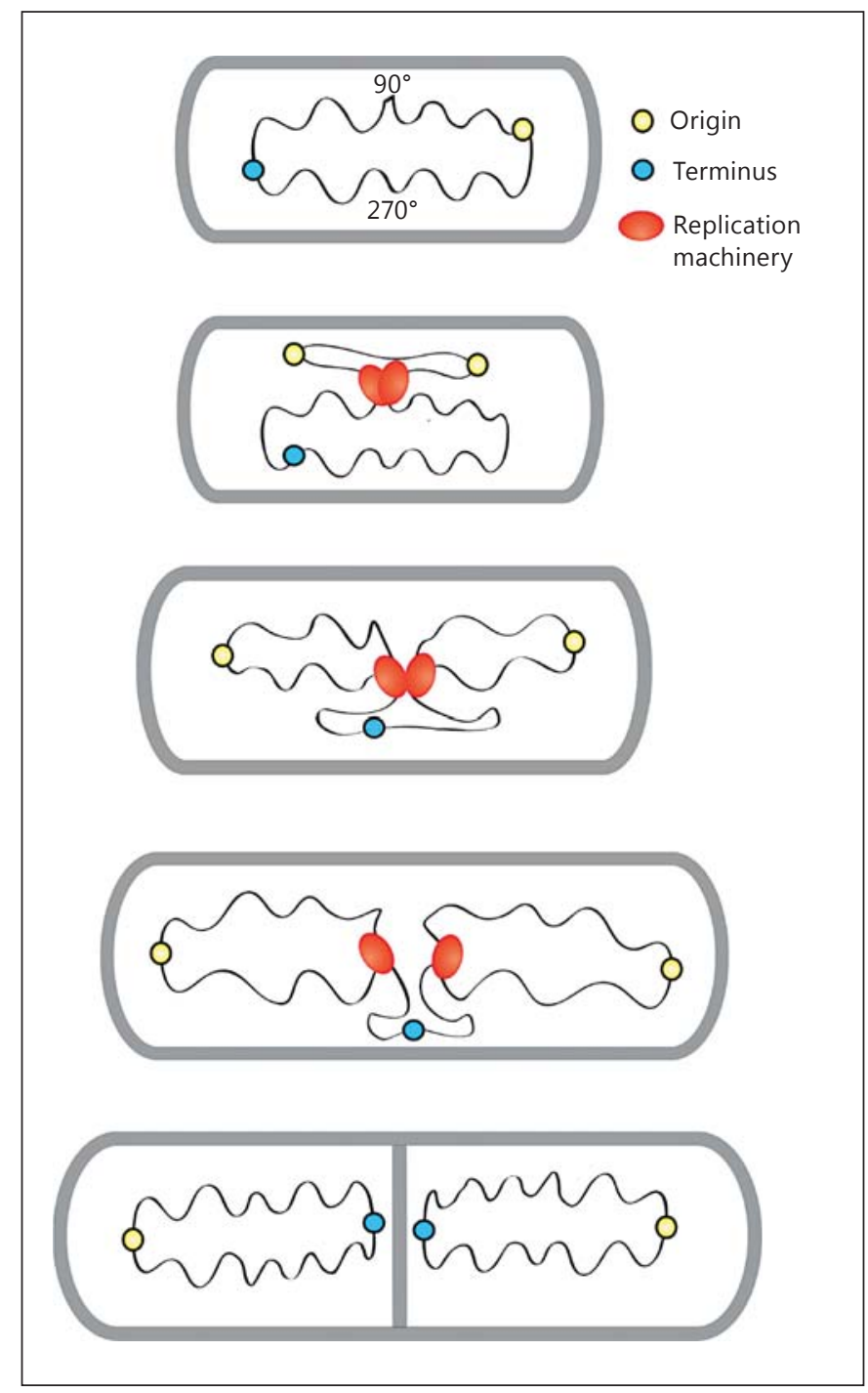

Fig. 2. Simplified model for the bacterial cell cycle under slowgrowth conditions based on $B$. subtilis (several aspects are different in e.g. E. coli or C. crescentus, and even in B. subtilis, the cell cycle is somewhat more complicated [Wang et al., 2014]). See text for details.

DNA gyrase, a type II topoisomerase, whereas excess negative supercoils are relaxed by topoisomerase I (Topo I). Upon thermal stress, the synthesis of one enzyme is reduced and that of the other increased. For instance, upon cold shock, more negative supercoils are needed to achieve strand opening, and thus the ratio of gyrase versus Topo I is increased [Lopez-Garcia and Forterre, 2000]. Thermophilic genomes are often positively supercoiled, mediated by the action of reverse gyrase, a type I topoisomerase [Valenti et al., 2011]. Positive or relaxed super-

Chromosome Architecture and

Segregation in Prokaryotic Cells coiling counteracts strand opening, thereby preventing spontaneous melting of DNA strands at high temperature. However, the superhelical state of bacterial and archaeal genomes is more complex, with different degrees of supercoiling coexisting, most likely due to the mentioned chromosome domains.

\section{Chromosome Arrangement and Segregation in Rod-Shaped and Curved Cells}

B. subtilis is a Gram-positive rod-shaped bacterium that has been used for many pioneering studies. It was also in this species that it was shown that the chromosome has a preferred arrangement, with origin regions being close to each cell pole [Webb et al., 1997], terminus region(s) close to the cell center, and $90^{\circ}$ and $270^{\circ}$ positions being positioned in between [Teleman et al., 1998] (fig. 1). It was later shown in much greater detail that several other bacterial genomes also have a defined 3-D layout, which however differs in arrangement (see below). The replication machinery in $B$. subtilis is positioned in the cell center in young cells, and during the later part of the cell cycle, the two replication forks move apart to the quarter sites of cells [Lemon and Grossman, 1998]. Even though closely adjacent to each other, replication forks can move apart and come back to the cell center, showing that the forks are not fixed to any specific site in the cell [Migocki et al., 2004]. Nevertheless, the relatively stationary positioning of replication forks implies that the chromosome moves through the cell center as it is being replicated, which was elegantly shown to be the case [Lemon and Grossman, 2000] (fig. 2). Soon after initiation of replication, origin regions move out towards opposite cell poles [Sharpe and Errington, 1998] through a mechanism that has not yet been elucidated. All other duplicated chromosome regions follow in a sequential manner such that replication and segregation occur concomitantly (fig. 2). Overall, the chromosome is arranged in a helical manner, and newly replicated DNA can be seen to emerge along a helical path from the cell center towards the poles [Berlatzky et al., 2008]. Spaces between the helical arms are filled at a later point of the replication center. A global helical architecture has also been seen for the E. coli chromosome [Fisher et al., 2013] and for Caulobacter [Umbarger et al., 2011], indicating that this may be a preferred way of folding a bacterial chromosome into a tubeshaped cell. Chromosome arrangement in B. subtilis is somewhat different from other bacteria: in slowly growing E. coli cells, the origin region and the terminus are in 
the cell center; the left arm of the chromosome is in one cell half, and the right arm is in the other [Niki et al., 2000; Wang et al., 2006] (fig. 1). Upon initiation of replication, the replication forks separate and move into opposite cell halves [Reyes-Lamothe et al., 2008]; from both forks, duplicated strands are moved into opposite cell halves, such that at the end of the cell cycle, the arrangement of chromosome arms (left in one cell half, right in the other) is identical in the sister cells. This is in contrast to the mirror image of duplicated chromosomes in e.g. B. subtilis (fig. 2) and in C. crescentus. However, during multifork replication, the orientation of the chromosome changes, and now chromosome arms lie next to each other along the long axis of the cells, rather than in opposite cell halves, and oldest replication forks are in the cell center, while younger forks are positioned outwards [Youngren et al., 2014]. This way, the chromosome is smoothly replicated and segregated via evenly spaced replication forks.

C. crescentus cells can only have two replication forks during the cell cycle, and thus are not able to perform multifork replication, like e.g. E. coli or B. subtilis. The origin of replication is stably attached to the cell pole at the beginning of the cell cycle; the terminus is at the other pole. The two arms of the chromosome lie side by side along the long axis of the cells, so the chromosome is condensed into an elongated donut (fig. 1). The replication machinery assembles at the polar origin, and slowly moves towards the opposite cell pole as it synthesizes new DNA strands [Jensen et al., 2001]. After duplication, one origin region is moved to the opposite pole, where it is again stably anchored. All duplicated regions are translocated towards opposite cell poles, into a position that mirrors the arrangement of the chromosome in the mother cell [Viollier et al., 2004]. Thus, the bottle brush-like chromosome is also replicated and segregated in highly ordered manner.

In Myxococcus xanthus, chromosome arrangement is somewhat similar to that found in C. crescentus; however, origin regions are not attached to the poles, but are stably positioned at a defined subpolar location [Harms et al., 2013]. Two replication forks move gradually from one origin towards the other edge of the nucleoid, and one newly replicated origin is translocated across the cell like in Caulobacter. The fact that chromosomes have preferred 3 -D arrangements within cells in at least 5 bacterial species (the above-mentioned and $V$. cholerae [see the review by Ramachandran et al., pp. 360-370]) suggests that this is a common feature in many if not all bacteria. Interestingly, a modeling study has shown that based on self-avoidance of DNA, the specific positioning of just a few DNA loci (such as origin and terminus), plus the organization of the chromosome into topological domains by DNA compaction proteins, is sufficient to generate a linear arrangement of the chromosome along the cell axis [Buenemann and Lenz, 2010]. Thus, geometric cues can largely shape a chromosome, taking advantage of intrinsic properties of DNA. For Caulobacter, polymeric PopZ protein mediates the stable attachment of origins to the old cell pole, and later in the cell cycle to opposite poles [Bowman et al., 2008; Ebersbach et al., 2008; Schofield et al., 2010]. ParB protein acts as an adaptor between origin regions and the polar anchor protein, which in $V$. cholerae is called HubP [Yamaichi et al., 2012]. In Corynebacterium glutamicum, which grows through the addition of new cell wall material at the cell pole, rather than at the lateral cell membrane like non-Actinomycete rod-shaped cells, origins are anchored to the poles via DivIVa, a protein that recognized negative membrane curvature [Lenarcic et al., 2009; Ramamurthi and Losick, 2009], and the adaptor again is a ParB protein [Donovan et al., 2012]. Thus, there are landmark proteins in bacteria that provide attachment points for specific chromosome regions and thereby help ordering the genome in 3-D. In bacteria such as E. coli and B. subtilis, chromosome origins are not firmly anchored to the cell envelope (except during sporulation in B. subtilis, where origins are placed at the cell poles via two dedicated proteins, RacA and DivIVa [Ben-Yehuda et al., 2003; Wu and Errington, 2003]), nor is any other site of the chromosome known to be positioned via a geometric cue from the cell envelope (fig. 1), so it remains to be seen how chromosome arrangement is achieved and maintained in these cases.

\section{Proteins Involved in Chromosome Segregation and Maintenance of Its 3-D Architecture}

DNA polymerase can contribute to an initial segregation of DNA because it is a powerful motor and, at least in case of B. subtilis, remains at its position for a long time during the cell cycle (fig. 1) [Lemon and Grossman, 1998]. However, DNA will bend at length exceeding $100 \mathrm{bp}$, so DNA cannot be pushed over a long distance, certainly not from the cell center to the poles in a 2- to $4-\mu \mathrm{m}$ cell. RNA polymerase generates strong pushing forces on the transcribed DNA, when the enzyme is immobilized. It has been proposed that RNA polymerase may contribute substantially to chromosome segregation. Evidence for this is seen from experiments showing that inhibition of transcription leads to a defect in segregation [Dworkin 
and Losick, 2002; Kjos and Veening, 2014] (under these experimental condition, transcription inhibition did not affect replication). The transcription bias of genes, facing away from oriC, and the fact that many origin-proximal genes are heavily transcribed may cause translocation of DNA towards the poles, when the many RNA polymerases (being very large enzyme complexes) act simultaneously on the DNA template, being coordinated in their movement.

Proteins playing an important role in chromosome dynamics are:

(a) NAPs, which form a variety of homo- or heterodimers, are abundant and influence chromosome structure, transcription and translation in various ways; they are covered in two reviews in this issue [Dorman, pp. 316331; van de Valk et al., pp. 344-359].

(b) ParA (Walker A ATPases) and ParB proteins, which are also important partitioning factors for many plasmids [Gerdes et al., 2004]. In some species, ParA and ParB are essential for segregation (and thus for viability), while in others, only mild segregation and/or replication defects occur upon deletion of parA or parB, which are always in an operon structure. ParA appears to act as a diffusion ratchet, providing directionality for the movement of replicated DNA segments from one cell half into the other half.

(c) SMC (structural maintenance of chromosomes) and the related MukB proteins, huge (50-nm-long) and widely conserved ring-like ATPases, whose absence in $B$. subtilis leads to severely impaired growth that is entirely blocked above $23^{\circ} \mathrm{C}$ in rich medium, and to the generation of about 15\% DNA-free (anucleate) cells [Kleine Borgmann and Graumann, pp. 384-395, and Rybenkov et al., pp. 371-383].

(d) Topoisomerases I, II (DNA gyrase) and IV, which are important for proper DNA supercoiling and resolution of disentangled chromosomes at the end of replication (fig. 3) [Muskhelishvili and Travers, pp. 332-343].

(e) DNA translocases (FtsK/SpoIIIE like) [discussed in the review by Crozat et al., pp. 396-408] which move DNA left at the division plane into future daughter cells (as ATP-driven hexameric ring motors; fig. 3), and also help position chromosome terminus regions, to facilitate or even activate the work of

(f) proteins achieving chromosome dimer resolution (e.g. Xer proteins). $10-15 \%$ of cells undergo an unequal number of recombination events between duplicated sister chromosomes, and are left with a single dimeric chromosome (fig. 3). Usually, two dedicated recombinases act at the dif site, positioned at the termini, to mediate resolu-

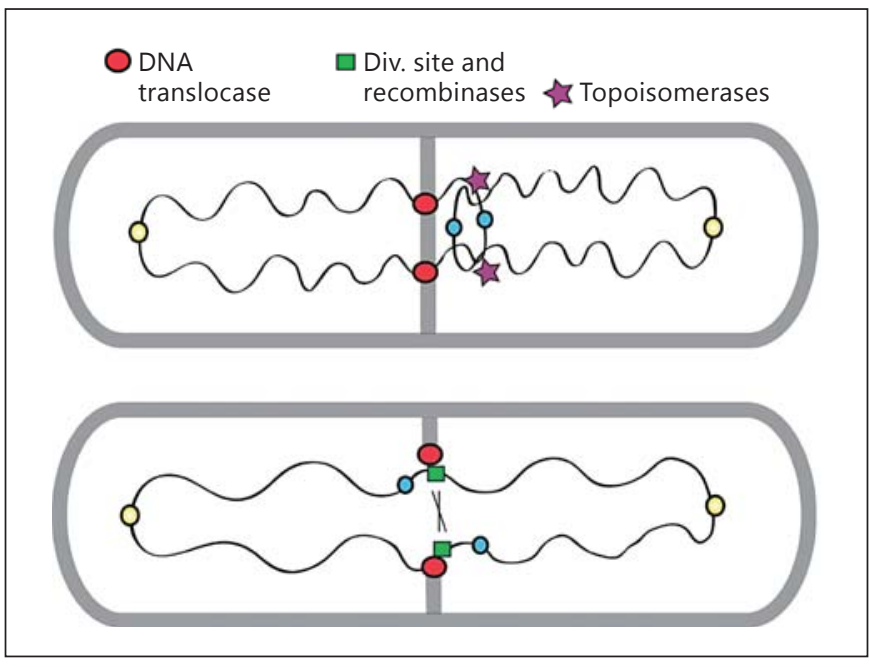

Fig. 3. Upper panel: segregation is coupled to cell division through DNA translocases, which move DNA away from the central division plane, or move DNA through the closed septum; for more details, see Kaimer and Graumann [2011]. Final steps of segregation also include the decatenation of intertwined sister chromosomes through type II topoisomerases (mainly topo IV, gyrase appears to be important at all times for disentanglement). Lower panel: resolution of chromosome dimers.

tion of chromosome dimers [see the review by Crozat et al., pp. 396-408].

It is of note that the absence of several proteins involved in DNA repair via homologous recombination also leads to segregation defects [Carrasco et al., 2004]. Thus, removal of recombination intermediates arising during DNA replication, likely based on stalling of replication forks, which occurs at a considerable rate during each cell cycle, is important for proper chromosome segregation.

\section{Segregation in Round and Oval Cells}

Few groups have taken up the task to answer the important biological question of how a round cell knows where to separate its chromosomes before cytokinesis has been commenced, and thus the division plane has been established. This is especially interesting in cells that change the plane of division each time following one cell cycle, such as Staphylococcus aureus. In contrast to most rod-shaped cells, where an $s m c(m u k B)$ deletion has a very severe segregation and growth defect, the deletion of $s m c$ in S. aureus or Streptococcus pneumoniae has a mild effect, such that the growth rate of mutant cells is not affected. 
However, when a DNA translocase is deleted in addition to $s m c$, chromosome segregation becomes much more inefficient, and ceases at high temperatures [Yu et al., 2010]. Thus, the translocation of DNA away from the closing (or even closed) division septum through the hexameric translocase rings plays an important role in a coccus. Interestingly, sublethal concentrations of transcription inhibitors lead to a considerable partitioning defect, while it leaves initial separation of origin regions largely unaffected, reminiscent of the effect seen in B. subtilis. Deletion of the transcription-processivity factor GreA also leads to the formation of an increased number of anucleate cells. Moreover, smc and parB mutant Streptococci are hypersensitive to transcription inhibitors, and the deletion of greA and $s m c$ is conditionally lethal [Kjos and Veening, 2014]. These experiments show that transcription (or a transcription-related factor) plays an important role in the separation of the bulk of the chromosome, and support the idea that RNA polymerase may play an additional motor-like function in chromosome segregation in bacteria.

\section{Segregation in Cells with Multiple Chromosome Copies: Cyanobacteria}

Cyanobacteria are a fascinating phylum within the bacteria, which for our purpose have one striking feature: many (if not most) species contain multiple chromosome copies, even under slow growth conditions. Thus, besides the ability to perform oxygenic photosynthesis and to undergo multiple different differentiation processes (formation of acinetes, heterocysts and hormogonia), these organisms are polyploid. In fact, many if not most bacterial species are polyploid, which is discussed in the review by Soppa [pp. 409-419].

In an initial study, the unicellular cyanobacterium Synechocystis sp. was used to address the question if multiple chromosomes (Synechocystis has about 10 copies during exponential growth) are distributed into future daughter cells at random, or in a defined, active manner. Synechocystis cells are round and can grow via the utilization of externally supplied glucose, and thus have a robust cell cycle when they do not oscillate between light and dark phases. Staining of the DNA showed that in contrast to $B$. subtilis, in which daughter cells receive DNA with an average deviation of $6 \%$ (which is expected to be experimental error), Synechocystis daughters show $15 \%$ average difference, with extreme cases in which one daughter can receive up to 2.5 times more DNA than the other cell. Thus, chromosome segregation seems to be less stringent in this cyanobacterium than in B. subtilis [Schneider et al., 2007]. A second study employed a more refined method to determine segregation in the filamentous species Anabaena sp., using an adapted parS/GFP-ParB system. It was observed that differences in fluorescence between integrated GFP-ParB spots in neighboring daughter cells are significantly larger than the variation in intensity of soluble GFP expressed between the cells, also suggesting some degree of randomness in the segregation process in a second cyanobacterium [Hu et al., 2007]. However, two studies of the rod-shaped Synechocystis elongatus revealed an astonishing degree of spatial organization of the multiple chromosomes: when origin regions were marked with a tetO/ TetR-CFP array and terminus regions with a lacO/LacIYFP system, the two signals were mostly observed in pairs, rather than randomly distributed, indicating that each chromosome occupies a certain territory. More intriguingly, at a certain time during the cell cycle, origin regions moved to become relatively evenly spaced along the long axis of cells, visually aligned in a straight line. Thus, chromosomes showed a high degree of spatial distribution, which gives rise to a rather even and accurate distribution of chromosomes before and after division. Interestingly, in mutant cells in which the septum is misplaced at various positions along the longitudinal axis of the cells, rather than precisely at mid cell, chromosomes are segregated unequally. Thus, there does not appear to be an active segregation machinery, but accurate chromosome positioning prevents unequal sister chromosome inheritance. Segregation of duplicated origin regions (and thus presumably their replication) was found to be asynchronous between the chromosomes, and copy number could vary considerably before or after division, suggesting that replication/segregation and cell division are not strongly coordinated. Indeed, a single replication machinery was observed in cells at a given time, such that only one or a subset of chromosomes is replicating at any given time. As this cyanobacterium appears to use chromosome alignment instead of active segregation, as well as ploidity (average of 4-5 chromosome copies), no need for a segregation/cytokinesis checkpoint would exist, and replication can also be nonstringently initiated.

Thus, cyanobacteria are a valuable system to study the segregation of multiple chromosomes, and can also be used to address an even more intricate question: how is chromosome segregation altered and arranged with cell branching? Type V cyanobacteria form cell branches and thus change the plane of chromosome segregation. The question of how the segregation mechanism is rearranged is a fascinating one. 


\section{Segregation in Cells with Two or More Different Chromosomes: V. cholerae}

$V$. cholerae has two distinct chromosomes, which are segregated by two different mechanisms [Fogel and Waldor, 2005; Srivastava et al., 2007], one involving a ParAB mechanism [Fogel and Waldor, 2006], and another yet unknown mechanism. That being stated, there is an excellent review on this interesting question of how to segregate different chromosomes in this issue of Journal of Molecular Microbiology and Biotechnology [see the review by Ramachandran et al., pp. 360-370].

\section{Chromosomes in Streptomyces}

Streptomycetes belong to the high GC Gram positives, also called Actinobacteria, which grow in a fungus-like apical manner, and have many other interesting properties. Streptomycetes form branched mycelia, and the model organism S. coelicolor has a linear chromosome, whose ends are held together by a dedicated protein, such that genetically, it behaves like a circular chromosome [Yang and Losick, 2001]. Its segregation is extremely sloppy: chromosomes are not condensed into nucleoids, but are unevenly distributed along the hyphae. This is in tune with cell division, which occurs infrequently and at irregular positions along the syncytia. In fact, $S$. coelicolor can even grow in the absence of FtsZ, i.e. the central player in cell division, as one large super-bacterium [McCormick et al., 1994]. In contrast, when nutrients are depleted and Streptomycetes initiate the formation of hyphae that grow into the air, for the ultimate formation of exospores, chromosome arrangement and segregation become highly ordered and coordinated: at the tip of aerial mycelium, about 20 or so discrete, orderly spaced nucleoids are formed, partially due to the function of ParAB, SMC, topoisomerase I and DNA translocase proteins [Dedrick et al., 2009; Jakimowicz et al., 2005; Szafran et al., 2013], such that synchronous septation between nucleoids gives rise to forespores, which upon maturation of the cell walls are released as dormant spores.

\section{Chromosomes in Spirochaetes and in Planctomycetes}

Spirochaetes form a phylum within the bacteria, and have an unusual cell structure: they contain their flagella within the periplasmic space and are highly helical. Fla- gellar rotation leads to a cork screw-like movement, which allows cells to move through viscous environments, and many pathogenic species use this property to squeeze between cells and thereby invade tissue. Borrelia hermsii contains 13-18 chromosomes and many plasmids, some of which are linear. The DNA is distributed through most of the cell's length [Kitten and Barbour, 1992]. In Treponema denticola, DNA is distributed throughout the cells. However, in Treponema cells lacking the filament-forming protein CftA, DNA is highly condensed, and cells show a chaining phenotype [Izard et al., 2001]. It is still unclear how CftA affects chromosome structure and cell division. It would be wonderful if better genetic tools were available for this group of bacteria to gain more insight into their cell cycle.

Planctomycetes belong to the Planctomycetes-Verrucomicrobia-Chlamydiae superphylum, which contains many most unusual species. Starting with the fact that many of these bacteria lack an FtsZ gene for cell division [van Niftrik et al., 2009], which is otherwise present in almost all bacteria and a majority of archaea, they provide many examples of unusual cell shapes and cell division types, including yeast-like budding [Lee et al., 2009]. Many Planctomycetes have a highly unusual cell architecture [Jogler et al., 2011], the details of which are still under debate. Some studies indicate the presence of additional intracellular membranes, which are not connected to the cell membrane. This is well established for the anamoxosomes, in which the unusual anamox reaction (generation of ammonia and nitrate in parallel from nitrogen) is driven by special enzymes, and which contain unusual ladderane lipids in the membrane that tightly seal off the reaction, which includes toxic intermediates [van Niftrik et al., 2009]. The existence of a nuclear-like envelope around the DNA has been reported, but was recently disputed, and instead, a highly invaginated cell membrane has been found for one species using cryo-electron tomography [Santarella-Mellwig et al., 2013]. Gemmata obscuriglobus has a highly amorphous nucleoid, or several nucleoids, in between which invaginated membranes can be found. DNA is translocated into the bud some time after its growth has been initiated [Lee et al., 2009], suggesting that an active transport mechanism exists. Genetic tools for relevant species have been established, so descriptions on the detailed structure of Planctomycetes and their cell cycle can hopefully be read by the community in the near future. 


\section{Chromosomes in Archaea}

Studies on archaeal chromosomes and their segregation are still scarce. The archaeon Halobacterium salinarum can be synchronized, and its study revealed that two SMC-like proteins, whose overproduction or depletion led to a delay in cell division, are only expressed late in the cell cycle [Herrmann and Soppa, 2002]. Thus, cell cycle proteins are also transcriptionally regulated in some archaea, like in Caulobacter, and contrarily to E. coli or B. subtilis, where all or most of these proteins are constitutively being produced throughout the cell cycle. H. salinarum DNA appears to be replicated in the cell center, where the DNA condenses early in the cell cycle, and sister chromosomes are separated towards quarter sites in a manner similar to the bacterial paradigm. However, archaeal chromosomes can differ notably from those of bacteria, e.g. by having several replication origins, by lacking replication stop (tus) sites, or by having histone-like proteins rather than NAPs. Further aspects of the archaeal replication/ segregation cycle are discussed by van der Valk et al. [pp. 344-359] and Samson and Bell [pp. 420-427].

\section{Active Motor versus Passive Segregation through Entropy}

Separate molecules and polymers tend to disentangle, especially if they have the same charge, based on diffusion. The idea that entropy plays a major role in the segregation of bacterial chromosomes has been introduced a long time ago. It can be shown by computer simulation that two fully replicated chromosomes segregate to opposite sides of a tube (i.e. rod-shaped cells) driven by (charge) repulsion of two self-avoiding polymers [Arnold and Jun, 2007; Jun and Mulder, 2006]. These simulations would argue that there is no need for an active machinery that pulls or pushes chromosome regions apart. In addition, it has been proposed that tethering of the chromosome to the membrane, which extends during the cell cycle, can also provide a force that aids in the separation of sister chromosomes. Such a tethering may be provided through a proposed mechanism called 'transertion', where the transcription of a gene coding for a membrane protein is directly coupled with the translation of its mRNA and the insertion of the growing peptide chain into the membrane [Woldringh and Nanninga, 2006]. Indeed, recent simulations show that membrane tethering strongly enhances the efficiency of complete separation of chromosomes driven by selfavoidance, and even more so if there is a polar gradient of
DNA attachment to the membrane [Di Ventura et al., 2013]. It has been proposed that MinD could set up such a gradient: the protein oscillates between the cell poles, driven by an ATPase cycle, and binds to DNA in vitro. In the absence of MinD, which helps positioning the cell division plane at the cell middle and binds to the cell membrane through an amphipathic helix, chromosome segregation has a visual defect. This occurs also when an arginine in MinD is mutated that is essential for DNA binding [Di Ventura et al., 2013]. The details of this DNA-membrane tether still need to be elaborated. In any event, it has been proposed that a purely entropy-driven separation of chromosomes is efficient only in separating the bulk of DNA, but energy drops sharply towards bulk separation, such that complete segregation will become inefficient. Moreover, the fact that chromosomes have highly ordered 3-D structures with preferred architecture argues against an 'entropy only'-driven process because separation through random diffusion cannot explain this degree of ordering. A more direct segregation mechanism has been demonstrated for $C$. crescentus and for $V$. cholerae: ParA ATPase protein localizes as a cloud-like structure from the polar origin to the opposite cell pole [Ptacin et al., 2010]. This structure retracts, and seemingly guides one duplicated origin region towards the other pole [Ptacin et al., 2014]. ParB induces ATPase activity in ParA, suggesting that origin-bound ParB protein (a specific DNA-binding protein) leads to the ParA retraction towards the opposite pole, mediating a diffusion ratchet-like movement of origins. ParA and ParB are essential in C. crescentus [Mohl and Gober, 1997], but not in V. cholerae [Kadoya et al., 2011], where ParA tethers origin regions of chromosome I to the poles, or in B. subtilis, where their deletion causes very mild segregation and replication defects [Ireton et al., 1994; Murray and Errington, 2008]. Moreover, E. coli cells do not possess genuine ParAB proteins, so it is clear that bacteria can use different segregation 'machineries' or mechanisms. Of note, low-copy plasmids in bacteria can use a ParAB-like partitioning system, an actin-like (ParM/ ParR) pushing mechanism [Becker et al., 2006; Gerdes et al., 2004], or a tubulin/FtsZ-like pushing apparatus [Larsen et al., 2007], revealing a marvelous array of different simple mitotic-like modules that can separate defined DNA segments in bacterial cells.

These beautiful reports notwithstanding, an active molecular machinery has been elusive in model bacteria such as B. subtilis and E. coli [for a discussion of possible mechanisms of segregation in E. coli, see the review by Messerschmidt and Waldminghaus, pp. 301-315], so in its absence, segregation must be assumed to be based on 
physico-/chemical mechanisms. I would like to speculate that in many bacteria, chromosome segregation has three layers: (a) several dedicated proteins or protein complexes (such as $\mathrm{SMC} / \mathrm{MukB}$ ) that compact DNA in a nonuniform manner ensure that separated chromosome regions remain in their given space [for more details, see the reviews by Kleine Borgmann and Graumann, pp. 384-395, and Rybenkov et al., pp. 371-383]; (b) sequence information on the chromosome sets up distinct domains via specific DNA binding proteins, possibly a topoisomerase gradient [see the review by Muskhelishvili and Travers, pp.332-343], and other architectural measures that shape the chromosome and aid in providing its 3-D arrangement within the cell, and (c) a passive segregation motor utilizes in part the power of DNA polymerase, which generates strong DNA pushing forces (over short distances), and entropy, to separate duplicated chromosome regions.
It will become apparent in this issue of Journal of Molecular Microbiology and Biotechnology that parts a and b have very interesting and intricate mechanisms, and that the field of bacterial chromosome structure and segregation is an exciting one promising to yield many more surprises in the future and finally insight into a very fundamental biological process.

\section{Acknowledgements}

Apologies to those colleagues whose work is not cited here due to limitations in space. Also, there are some topics relevant to chromosome structure and segregation that are not dealt with in great detail, but are covered in other parts of this special Journal of Molecular Microbiology and Biotechnology issue. This work has been supported by the LOEWE Centre SYNMIKRO of the state of Hessen and by the Deutsche Forschungsgemeinschaft.

\section{References}

Arnold A, Jun S: Time scale of entropic segregation of flexible polymers in confinement: implications for chromosome segregation in filamentous bacteria. Phys Rev E Stat Nonlin Soft Matter Phys 2007;76:031901.

- Becker E, Herrera NC, Gunderson FQ, Derman AI, Dance AL, Sims J, Larsen RA, Pogliano J: DNA segregation by the bacterial actin AlfA during Bacillus subtilis growth and development. EMBO J 2006;25:5919-5931.

-Ben-Yehuda S, Rudner DZ, Losick R: RacA, a bacterial protein that anchors chromosomes to the cell poles. Science 2003;299:532-536.

Berlatzky IA, Rouvinski A, Ben-Yehuda S: Spatial organization of a replicating bacterial chromosome. Proc Natl Acad Sci USA 2008; 105 : 14136-14140.

Bowman GR, Comolli LR, Zhu J, Eckart M, Koenig M, Downing KH, Moerner WE, Earnest T, Shapiro L: A polymeric protein anchors the chromosomal origin/ParB complex at a bacterial cell pole. Cell 2008;134:945-955.

-Buenemann M, Lenz P: A geometrical model for DNA organization in bacteria. PloS One 2010; 5:e13806.

-Carrasco B, Cozar MC, Lurz R, Alonso JC, Ayora $\mathrm{S}$ : Genetic recombination in Bacillus subtilis 168: contribution of Holliday junction processing functions in chromosome segregation. J Bacteriol 2004;186:5557-5566.

Dedrick RM, Wildschutte H, McCormick JR: Genetic interactions of $s m c$, fts $K$, and parB genes in Streptomyces coelicolor and their developmental genome segregation phenotypes. J Bacteriol 2009;191:320-332.

-Di Ventura B, Knecht B, Andreas H, Godinez WJ, Fritsche M, Rohr K, Nickel W, Heermann DW, Sourjik V: Chromosome segregation by the Escherichia coli Min system. Mol Syst Biol 2013;9:686.

Donovan C, Sieger B, Kramer R, Bramkamp M: A synthetic Escherichia coli system identifies a conserved origin tethering factor in Actinobacteria. Mol Microbiol 2012;84:105-116.

Dworkin J, Losick R: Does RNA polymerase help drive chromosome segregation in bacteria? Proc Natl Acad Sci USA 2002;99:14089-14094.

-Ebersbach G, Briegel A, Jensen GJ, Jacobs-Wagner C: A self-associating protein critical for chromosome attachment, division, and polar organization in Caulobacter. Cell 2008;134: 956-968.

Fisher JK, Bourniquel A, Witz G, Weiner B, Prentiss M, Kleckner N: Four-dimensional imaging of $E$. coli nucleoid organization and dynamics in living cells. Cell 2013;153:882-895.

Fogel MA, Waldor MK: Distinct segregation dynamics of the two Vibrio cholerae chromosomes. Mol Microbiol 2005;55:125-136.

Fogel MA, Waldor MK: A dynamic, mitotic-like mechanism for bacterial chromosome segregation. Genes Dev 2006;20:3269-3282.

- Gerdes K, Moller-Jensen J, Ebersbach G, Kruse T, Nordstrom K: Bacterial mitotic machineries. Cell 2004;116:359-366.

Graumann PL, Knust T: Dynamics of the bacterial SMC complex and SMC-like proteins involved in DNA repair. Chromosome Res 2009; 17:265-275.

Harms A, Treuner-Lange A, Schumacher D, Sogaard-Andersen L: Tracking of chromosome and replisome dynamics in Myxococcus xanthus reveals a novel chromosome arrangement. PLoS Genet 2013;9:e1003802.

Herrmann U, Soppa J: Cell cycle-dependent expression of an essential SMC-like protein and dynamic chromosome localization in the archaeon Halobacterium salinarum. Mol Microbiol 2002;46:395-409.

Hu B, Yang G, Zhao W, Zhang Y, Zhao J: MreB is important for cell shape but not for chromosome segregation of the filamentous cyanobacterium Anabaena sp. PCC 7120. Mol Microbiol 2007;63:1640-1652.

Ireton K, Gunther AD, Grossman AD: spo0J is required for normal chromosome segregation as well as the initiation of sporulation in Bacillus subtilis. J Bacteriol 1994;176:5320-5329.

- Itaya M, Tsuge K, Koizumi M, Fujita K: Combining two genomes in one cell: stable cloning of the Synechocystis PCC6803 genome in the Bacillus subtilis 168 genome. Proc Natl Acad Sci USA 2005;102:15971-15976.

Izard J, Samsonoff WA, Limberger RJ: Cytoplasmic filament-deficient mutant of Treponema denticola has pleiotropic defects. J Bacteriol 2001;183:1078-1084.

Jakimowicz D, Gust B, Zakrzewska-Czerwinska J, Chater KF: Developmental-stage-specific assembly of ParB complexes in Streptomyces coelicolor hyphae. J Bacteriol 2005; 187:3572-3580.

-Jensen RB, Wang SC, Shapiro L: A moving DNA replication factory in Caulobacter crescentus. EMBO J 2001;20:4952-4963.

Jogler C, Glockner FO, Kolter R: Characterization of Planctomyces limnophilus and development of genetic tools for its manipulation establish it as a model species for the phylum Planctomycetes. Appl Environ Microbiol 2011;77:5826-5829.

Jun S, Mulder B: Entropy-driven spatial organization of highly confined polymers: lessons for the bacterial chromosome. Proc Natl Acad Sci USA 2006;103:12388-12393.
Chromosome Architecture and

Segregation in Prokaryotic Cells
J Mol Microbiol Biotechnol 2014;24:291-300 DOI: $10.1159 / 000369100$ 
Kadoya R, Baek JH, Sarker A, Chattoraj DK: Participation of chromosome segregation protein ParAI of Vibrio cholerae in chromosome replication. J Bacteriol 2011;193:1504-1514.

-Kaimer C, Graumann PL: Players between the worlds: multifunctional DNA translocases. Curr Opin Microbiol 2011;14:719-725.

Kitten T, Barbour AG: The relapsing fever agent Borrelia hermsii has multiple copies of its chromosome and linear plasmids. Genetics 1992;132:311-324.

Kjos M, Veening JW: Tracking of chromosome dynamics in live Streptococcus pneumoniae reveals that transcription promotes chromosome segregation. Mol Microbiol 2014;91: 1088-1105.

Larsen RA, Cusumano C, Fujioka A, Lim-Fong G, Patterson P, Pogliano J: Treadmilling of a prokaryotic tubulin-like protein, TubZ, required for plasmid stability in Bacillus thuringiensis. Genes Dev 2007;21:1340-1352.

Le TB, Imakaev MV, Mirny LA, Laub MT: Highresolution mapping of the spatial organization of a bacterial chromosome. Science 2013 342:731-734.

Lee KC, Webb RI, Fuerst JA: The cell cycle of the planctomycete Gemmata obscuriglobus with respect to cell compartmentalization. BMC Cell Biol 2009; 10:4.

Lemon KP, Grossman AD: Localization of bacterial DNA polymerase: evidence for a factory model of replication. Science 1998;282:15161519.

Lemon KP, Grossman AD: Movement of replicating DNA through a stationary replisome. Mol Cell 2000;6:1321-1330.

Lenarcic R, Halbedel S, Visser L, Shaw M, Wu LJ, Errington J, Marenduzzo D, Hamoen LW: Localisation of DivIVA by targeting to negatively curved membranes. EMBO J 2009;28: 2272-2282.

Lopez-Garcia P, Forterre P: DNA topology and the thermal stress response, a tale from mesophiles and hyperthermophiles. Bioessays 2000;22:738-746.

-McCormick JR, Su EP, Driks A, Losick R: Growth and viability of Streptomyces coelicolor mutant for the cell division gene ftsZ. Mol Microbiol 1994;14:243-254.

-Merrikh H, Machon C, Grainger WH, Grossman $\mathrm{AD}$, Soultanas P: Co-directional replicationtranscription conflicts lead to replication restart. Nature 2011:470:554-557.

-Migocki MD, Lewis PJ, Wake RG, Harry EJ: The midcell replication factory in Bacillus subtilis is highly mobile: implications for coordinating chromosome replication with other cell cycle events. Mol Microbiol 2004;54:452-463.

Mohl DA, Gober JW: Cell cycle-dependent polar localization of chromosome partitioning proteins in Caulobacter crescentus. Cell 1997;88: 675-684.

- Murray H, Errington J: Dynamic control of the DNA replication initiation protein DnaA by Soj/ParA. Cell 2008;135:74-84.
Niki H, Yamaichi Y, Hiraga S: Dynamic organization of chromosomal DNA in Escherichia coli. Genes Dev 2000;14:212-223.

- Postow L, Hardy CD, Arsuaga J, Cozzarelli NR: Topological domain structure of the Escherichia coli chromosome. Genes Dev 2004;18 1766-1779.

Ptacin JL, Gahlmann A, Bowman GR, Perez AM, von Diezmann AR, Eckart MR, Moerner WE, Shapiro L: Bacterial scaffold directs pole-specific centromere segregation. Proc Natl Acad Sci USA 2014;111:E2046-E2055.

Ptacin JL, Lee SF, Garner EC, Toro E, Eckart M, Comolli LR, Moerner WE, Shapiro L: A spindle-like apparatus guides bacterial chromosome segregation. Nat Cell Biol 2010;12:791798.

Ramamurthi KS, Losick R: Negative membrane curvature as a cue for subcellular localization of a bacterial protein. Proc Natl Acad Sci USA 2009; 106:13541-13545.

Reyes-Lamothe R, Possoz C, Danilova O, Sherratt DJ: Independent positioning and action of Escherichia coli replisomes in live cells. Cell 2008;133:90-102.

-Santarella-Mellwig R, Pruggnaller S, Roos N, Mattaj IW, Devos DP: Three-dimensional reconstruction of bacteria with a complex endomembrane system. PLoS Biol 2013;11: e1001565.

-Schneider D, Fuhrmann E, Scholz I, Hess WR, Graumann PL: Fluorescence staining of live cyanobacterial cells suggest non-stringent chromosome segregation and absence of a connection between cytoplasmic and thylakoid membranes. BMC Cell Biol 2007;8:39.

Schofield WB, Lim HC, Jacobs-Wagner C: Cell cycle coordination and regulation of bacterial chromosome segregation dynamics by polarly localized proteins. EMBO J 2010;29:30683081.

Sharpe ME, Errington J: A fixed distance for separation of newly duplicated copies of oriC in Bacillus subtilis: implications for co-ordination of chromosome segregation and cell division. Mol Microbiol 1998;28:981-990.

- Srivastava P, Demarre G, Karpova TS, McNally J, Chattoraj DK: Changes in nucleoid morphology and origin localization upon inhibition or alteration of the actin homolog, MreB, of Vibrio cholerae. J Bacteriol 2007;189:7450-7463.

-Staczek P, Higgins NP: Gyrase and Topo IV modulate chromosome domain size in vivo. Mol Microbiol 1998;29:1435-1448.

Szafran M, Skut P, Ditkowski B, Ginda K, Chandra G, Zakrzewska-Czerwinska J, Jakimowicz D: Topoisomerase I (TopA) is recruited to ParB complexes and is required for proper chromosome organization during Streptomyces coelicolor sporulation. J Bacteriol 2013; 195:4445-4455.

Teleman AA, Graumann PL, Lin DCH, Grossman $\mathrm{AD}$, Losick R: Chromosome arrangement within a bacterium. Curr Biol 1998;8: 1102-1109.
Umbarger MA, Toro E, Wright MA, Porreca GJ, Bau D, Hong SH, Fero MJ, Zhu LJ, Marti-Renom MH, McAdams HH, Shapiro L, Dekker J, Church GM: The three-dimensional architecture of a bacterial genome and its alteration by genetic perturbation. Mol Cell 2011;44: 252-264.

Valenti A, Perugino G, Rossi M, Ciaramella M: Positive supercoiling in thermophiles and mesophiles: of the good and evil. Biochem Soc Trans 2011;39:58-63.

van Niftrik L, Geerts WJ, van Donselaar EG, Humbel BM, Webb RI, Harhangi HR, Camp HJ, Fuerst JA, Verkleij AJ, Jetten MS, Strous M: Cell division ring, a new cell division protein and vertical inheritance of a bacterial organelle in anammox planctomycetes. Mol Microbiol 2009;73:1009-1019.

-Viollier PH, Thanbichler M, McGrath PT, West L, Meewan M, McAdams HH, Shapiro L: Rapid and sequential movement of individual chromosomal loci to specific subcellular locations during bacterial DNA replication. Proc Natl Acad Sci USA 2004;101:9257-9262.

-Wang X, Liu X, Possoz C, Sherratt DJ: The two Escherichia coli chromosome arms locate to separate cell halves. Genes Dev 2006;20:17271731.

Wang X, Montero Llopis P, Rudner DZ: Bacillus subtilis chromosome organization oscillates between two distinct patterns. Proc Natl Acad Sci USA 2014;111:12877-12882.

Webb CD, Teleman A, Gordon S, Straight A, Belmont A, Lin DC-H, Grossman AD, Wright A, Losick R: Bipolar localization of the replication origin regions of chromosomes in vegetative and sporulating cells of $B$. subtilis. Cell 1997;88:667-674.

Woldringh CL, Nanninga N: Structural and physical aspects of bacterial chromosome segregation. J Struct Biol 2006;156:273-283.

-Wu LJ, Errington J: RacA and the Soj-Spo0J system combine to effect polar chromosome segregation in sporulating Bacillus subtilis. Mol Microbiol 2003;49:1463-1475.

- Yamaichi Y, Bruckner R, Ringgaard S, Moll A, Cameron DE, Briegel A, Jensen GJ, Davis BM, Waldor MK: A multidomain hub anchors the chromosome segregation and chemotactic machinery to the bacterial pole. Genes Dev 2012;26:2348-2360.

Yang MC, Losick R: Cytological evidence for association of the ends of the linear chromosome in Streptomyces coelicolor. J Bacteriol 2001;183:5180-5186.

Youngren B, Nielsen HJ, Jun S, Austin S: The multifork Escherichia coli chromosome is a selfduplicating and self-segregating thermodynamic ring polymer. Genes Dev 2014;28:7184.

Yu W, Herbert S, Graumann PL, Gotz F: Contribution of SMC (structural maintenance of chromosomes) and SpoIIIE to chromosome segregation in Staphylococci. J Bacteriol 2010; 192:4067-4073. 